\title{
Having a cluttered year from the coronavirus
}

\author{
Chang-Ju Kim (D) https://orcid.org/0000-0003-4749-5795
}

It can be said that 2020 was the year of the coronavirus. Coronavirus began to pandemic in China's Yuhan from the beginning of the year and hit the world, and the aftereffects are still ongoing, and the world has not escaped from the shock. It was the year 2020 that started with the coronavirus and ended with the coronavirus.

Many of the papers submitted to the Journal of Exercise Rebabilitation (JER) were research on exercise under the coronavirus epidemic. However, these papers were not research on exercise under the special conditions of coronavirus outbreaks. It is just setting the environment of coronavirus to the content of general exercise. Many papers on coronavirus are being submitted to journals around the world. However, JER has no papers on coronavirus other than editorial. Although coronavirus is outbreak, I don't think it is right to blame all situations where it is difficult to exercise. Exercise performance is not very limited by coronavirus. There is a need for research into the mechanism of how exercise affects the human body. Although the effectiveness of exercise has been largely confirmed in many diseases, research on the mechanism is insufficient. Among the papers submitted to JER, there are not many studies on the mechanism. We look forward to submitting many papers to JER on the effects of exercise on the body and the mechanisms of various diseases.

Due to coronavirus, academic conferences could not be held. The
Korean Society of Exercise Rehabilitation also held a dinner meeting on November 6th, as the Spring and Autumn conferences were not held in 2020. Through this meal meeting, we tried to promote communication among members. Although only dinner meeting was held this year, I hope to hold normal academic conferences in 2021.

The coronavirus has had a very profound impact on people around the world, and it has changed the way people live. Lectures and meetings were also held through the Internet, and many stores were closed due to deficits. The era of coronavirus that has made our lives uncomfortable and has caused tremendous economic losses. I hope that these chaotic times will pass quickly and normal life will resume.

\section{CONFLICT OF INTEREST}

No potential conflict of interest relevant to this article was reported.

President of the Korean Society of Exercise Rehabilitation Department of Physiology, College of Medicine Kyung Hee University 26 Kyungheedae-ro, Dongdaemun-gu, Seoul 02447, Korea E-mail: changju@khu.ac.kr 